\title{
LA NOBLEZA NAPOLITANA EN LA MONARQUÍA HISPANA: EL PARLAMENTO DEL REINO DE NÁPOLES (1598-1642)
}

\author{
Giuseppe Mrozek Eliszezynski \\ (Universidad “G. D’annunzio" di Chieti-Pescara)
}

\section{RESUMEN}

El artículo tiene como objetivo presentar algunos elementos de reflexión derivados de un estudio más general, centrado en la conducta política de la aristocracia napolitana durante el siglo XVII. El análisis de las sesiones del Parlamento del reino de Nápoles, convocadas hasta 1642, es una excelente herramienta para investigar cómo actuaron las principales familias del reino durante varias décadas, en particular en momentos de gran incertidumbre: en la sucesión del rey fallecido a su heredero o incluso en la transición del gobierno de un ministro-favorito a otro. La conexión con la lucha política que se desarrollaba en la corte de Madrid y las relaciones entre los aristócratas napolitanos y los virreyes que eran expresión del gobierno del valido del momento son, de hecho, elementos centrales dentro de este tema.

PALABRAS CLAVE: Nápoles; Parlamento; Virreyes; Nobleza; Validos.

\section{NEAPOLITAN NOBILITY IN THE SPANISH MONARCHY: THE PARLIAMENT OF THE KINGDOM OF NAPLES (1598-1642)}

\begin{abstract}
The article aims to present some reflections from a more general study focused on the political conduct of the Neapolitan aristocracy during the seventeenth century. The sessions' analysis of the Parliament of the Kingdom of Naples, convened until 1642, is an excellent tool to investigate how the leading families of the Kingdom have acted for decades, in particular during moments of great uncertainty: in the succession from a deceased king to his heir or even in the transition from the government of a minister-favorite to another. The connection with the political fight of the Madrid court and the relations between the Neapolitan aristocrats and the viceroys who represented the policy developed by the favorite of that moment are, in fact, central elements within this theme.
\end{abstract}

KEY WORDS: Naples; Parliament; Viceroys; Nobility; Minister-Favorites. 
El diálogo y, a menudo, el choque entre la aristocracia del reino de Nápoles y los virreyes que llegaban al sur de Italia para representar al Rey de España son dos elementos básicos y siempre presentes en la historia bicentenaria de la dominación ibérica sobre la Península italiana. Durante el período del valimiento, o sea durante los gobiernos del duque de Lerma, del conde-duque de Olivares y de don Luis de Haro, esta tendencia se hizo aún más delicada, porque la pertenencia o no de la nobleza napolitana al abigarrado frente que se oponía a la política desarrollada por los favoritos de Felipe III y Felipe IV es esencial para la comprensión de los acontecimientos de aquellos años, a partir de los numerosos momentos de tensión que se registraron en la primera mitad del siglo XVII y que culminaron en la revuelta de 1647-1648. Para reforzar y confirmar amistades y alianzas, a menudo se recurría al intercambio de regalos y objetos preciosos, y los virreyes implicaban en su política cultural a personalidades afines a ellos no solo desde el punto de vista político, sino también en intereses y gustos artísticos y literarios. El propósito de esta intervención es, por lo tanto, presentar algunos elementos de reflexión dentro de un camino a largo plazo y de una investigación más amplia que estoy realizando en los últimos meses. Lo que propongo poner de manifiesto es cómo sería posible identificar, a pesar de que hasta ahora la historiografía sobre el reino de Nápoles no lo haya hecho, una conducta política bien definida por parte de las principales familias aristocráticas napolitanas, que con el paso de las generaciones mantuvieran una cierta línea de acción y fuertes lazos, a veces de alianza y algunas veces de oposición, con la aristocracia española de la cual los virreyes eran representantes ideales. ${ }^{1}$

Una de las mejores herramientas para entender el comportamiento de los nobles napolitanos durante el período en cuestión es el estudio de las actas de las sesiones del Parlamento, la asamblea que fue convocada, hasta 1642, para aprobar el denominado donativo, un impuesto directo que pesaba sobre los súbditos napolitanos del Rey Católico y cuya cantidad se había estabilizado, a lo largo de los años, en la cifra de 1.200.000 ducados. Además de esa suma, el Parlamento a menudo autorizó otras

* Abreviaturas: BNE (Biblioteca Nacional de España); BNN (Biblioteca Nazionale di Napoli); BSNSP (Biblioteca della Società Napoletana di Storia Patria); DBI (Dizionario Biografico degli Italiani).

${ }^{1} \mathrm{La}$ investigación sobre la aristocracia napolitana se ha centrado hasta ahora en otros aspectos, como la cultura y el modo de vida nobiliario: véanse, por ejemplo, Maria Antonietta Visceglia, Il bisogno di eternità. I comportamenti aristocratici a Napoli in età moderna (Nápoles: Guida, 1988); Giovanni Muto, “«I segni d'honore». Le rappresentazioni delle dinamiche nobiliari a Napoli in età moderna", en Signori, patriz̨i, cavalieri nell'età moderna, ed. Maria Antonietta Visceglia (Roma-Bari: Laterza, 1992), 171-192; Maria Antonietta Visceglia, Identità sociali. La nobiltà napoletana nella prima età moderna (Milán: Unicopli, 1998). Objeto de interés ha sido también la gestión patrimonial y financiera de familias o individuos, como por ejemplo en Francesco Dandolo y Gaetano Sabatini, Lo Stato feudale dei Carafa di Maddaloni. Genesi e amministrazione di un ducato nel regno di Napoli (secc. XV-XVIII) (Nápoles: Giannini, 2009). Otros estudios se han dedicado a la conducta política de unos clanes en momentos específicos de la historia del Mezzogiorno: véanse, en particular los estudios de Giulio Sodano, Da baroni del Regno a Grandi di Spagna. Gli Acquaviva d'Atri: vita aristocratica e ambizioni politiche (secoli XV-XVIII) (Nápoles: Guida, 2012), y "Le aristocrazie napoletane", en Il Regno di Napoli nell'età di Filippo IV (1621-1665), eds. Giovanni Brancaccio y Aurelio Musi (Milán: Guerini e Associati, 2014), 131-176. Hasta el momento, sin embargo, no hay una visión general del posicionamiento y de las estrategias políticas de la aristocracia del reino durante la dominación española. 
formas de tributación extraordinaria, pidiendo a cambio al virrey, y a través de él al rey, el cumplimiento de una serie de gracias y privilegios, debiéndose entender por estos términos no solo solicitudes y concesiones una tantum, sino también cambios en las leyes y regulaciones del reino. La historiografía ha disminuido por mucho tiempo la importancia del Parlamento napolitano, comparándolo no solo con el ejemplo inglés, sino también con el ejemplo siciliano más cercano. ${ }^{2}$ A pesar de las dificultades relacionadas con la pérdida, durante la Segunda Guerra Mundial, de la documentación original de las reuniones, el estudio de las copias y de los fragmentos conservados de las diferentes sesiones del Parlamento napolitano ayuda a entender la posición de la aristocracia napolitana con respecto a las propuestas de los diferentes virreyes y los validos de los cuales a menudo eran poderosos y fieles aliados. También hay que considerar cómo la aristocracia napolitana, lejos de ser un bloque monolítico, ni un grupo unido en defensa de sus privilegios, se dividió internamente tanto en varias facciones como en dos grupos tradicionales: el baronaggio (la nobleza feudal fuerte especialmente en las provincias) y los Seggi (la nobleza urbana, enraizada en la capital napolitana y tradicionalmente desplegada en cinco grupos, o sea los Seggi $)$. Con los años, las principales familias feudales entraron en las filas de la nobleza urbana, y los Seggi llegaron a desempeñar un papel dominante, plenamente confirmado por la figura del sindaco (alcalde): este era el presidente del Parlamento (encargado de presentar al virrey los resultados de la reunión parlamentaria), y representaba a su vez a uno de los cinco Seggi nobles de Nápoles. ${ }^{3}$ Todo esto se desarrolló a pesar de que la capital, por

2 La supuesta poca relevancia histórica del Parlamento napolitano ya había sido subrayada por Benedetto Croce, Storia del Regno di Napoli (Bari: Laterza, 1925), y reafirmada en el ensayo de la hija del filósofo, Elena Croce, "I parlamenti napoletani sotto la dominazione spagnola", Archivio storico per le province napoletane 61 (1936): 341-379. Esta condena, ya evidenciada por Giuseppe Galasso en Mezzogiorno medievale e moderno (Turín: Einaudi, 1965), 195, ha sido solo parcialmente superada por la historiografía que ha abordado el tema con posterioridad, a menudo considerándolo dentro de la cuestión más general de la importancia de los Parlamentos en la Italia española: véanse los estudios de Antonio Marongiu, Il Parlamento in Italia nel medioevo e nell'età moderna (Milán: Giuffrè, 1962); Francesco Caracciolo, "Il Parlamento del Regno di Napoli durante la dominazione spagnola", Quaderni contemporanei 4 (1971): 21 58; Guido D’Agostino, Parlamento e società nel Regno di Napoli, secoli XV-XVII (Nápoles: Guida, 1979). En tiempos más recientes, ha vuelto sobre el tema Francesco Benigno, "Persistere, resistere: Parlamenti italiani e Monarchia degli Asburgo", en Id., Favoriti e ribelli. Stili della politica barocca (Roma: Bulzoni, 2011), 147-163. Por último se señala, aunque se refiera al período del dominio aragonés, el estudio de Elisabetta Scarton y Francesco Senatore, Parlamenti generali a Napoli in età aragonese (Nápoles: Federico II University Press, 2018).

${ }^{3}$ Sobre los Seggi de Nápoles (cinco nobiliares, o sea Nido, Capuana, Porto, Portanova y Montagna, y uno en representación del Pueblo de Nápoles) y la idea de nobleza que representaban, véanse las obras fundamentales de Camillo Tutini, Dell'origine e fundation de' Seggi di Napoli, del tempo in che furono instituiti e della separation de' nobili dal popolo (Nápoles: Beltrano, 1644); y Francesco Capecelatro, Origine della città e delle famiglie nobili di Napoli (Nápoles: Gravier, 1769). El poder de las principales familias napolitanas, que se extendía desde la capital a las distintas provincias del reino, se expresa también en diversas colecciones: Scipione Ammirato, Delle famiglie nobili napoletane (Florencia: Marescotti, 1580-1651); Filiberto Campanile, L'Armi overo insegne de' Napoli (Nápoles: Longo, 1610); Carlo De Lellis, Discorsi delle famiglie nobili del Regno di Napoli (Nápoles 1654-1671); Biagio Aldimari, Memorie historiche di diverse famiglie nobili, cosi napolitane come forastiere, cosi vive come spente, con le loro arme; e con un trattato dell'arme in generale (Nápoles: Raillard, 1691); BNE, Mss. 8415: Notizie d'alcune famiglie popolari della città e Regno di Napoli 
privilegio antiguo y consolidado, estaba exenta de impuestos. Las que perdieron progresivamente representatividad y poder fueron, en cambio, las ciudades demaniali (estatales), también teóricamente presentes en el Parlamento pero en realidad representadas por procuradores que, en la mayoría de los casos, eran aristócratas. Para el Pueblo de Nápoles quedaban dos de los veinticuatro miembros que formaban la llamada Deputazione delle grazie, a saber, el órgano compuesto por doce miembros de la nobleza feudal y doce procedentes de los seis Seggi de la capital (dos por cada uno de los cinco Seggi nobles más el popular) a cargo de presentar al virrey las solicitudes de gracias y privilegios.

Durante el valimiento del duque de Lerma (1598-1618), el virreinato de Nápoles fue ejercido por personajes de la primera nobleza castellana y claramente relacionados con el valido y su facción por lazos familiares y/o por intereses políticos comunes. ${ }^{4}$ Tras el breve gobierno del VI conde de Lemos (1599-1601), cuñado y al mismo tiempo consuegro de Lerma, que murió durante su cargo y fue sustituido, hasta 1603, por su segundo hijo Francisco de Castro (futuro virrey de Sicilia), ${ }^{5}$ llegó el largo gobierno del conde de Benavente (1603-1610), otro fiel intérprete de la política lermista. Pero fue el siguiente virrey, el VII conde de Lemos (1610-1616), quien representó mejor que nadie la influencia del valido de Felipe III en el gobierno de Nápoles. Sobrino, como hijo de una de las hermanas de Lerma, pero también yerno del valido, porque se había casado con una de las hijas del mismo Lerma, Lemos disfrutó no solo las ventajas de una doble conexión familiar, sino también la estimación total del favorito, hasta al punto que en la corte de Madrid muchos vieron en él, y no en el primogénito de Lerma, el duque de Uceda, el verdadero heredero del valimiento. ${ }^{6}$ A la luz de estas consideraciones, se entiende fácilmente cómo el gobierno del VII conde de Lemos haya sido tradicionalmente leído junto con el de su sucesor, el duque de Osuna (1616-1620). ${ }^{7}$ Estos dos grandes aristócratas simbolizaban los dos grupos en

divenute per ricchezza e dignità riguardevoli, d'incerto autore, año 1693, ff. 107v-110v; Giuseppe Recco, Notizie di famiglie nobili ed illustri della città e Regno di Napoli (Nápoles: Parrino, 1717); Berardo Candida Gonzaga, Memorie delle famiglie nobili delle province meridionali d'Italia, 6 vols. (Nápoles: De Angelis e figlio, 1875-1879).

${ }^{4}$ Francesco Benigno, L'ombra del re. Ministri e lotta politica nella Spagna del Seicento (Venecia: Marsilio, 1992), 37-94; Giuseppe Galasso, Il Mezzogiorno spagnolo e austriaco, en Storia d'Italia, ed. Giuseppe Galasso (Turín: Einaudi, 2006), vol. XV, t. II, 1494-1622, 882-1050. Me permito también reenviar a Giuseppe Mrozek Eliszezynski, Bajo acusación: el valimiento en el reinado de Felipe III. Procesos y discursos (Madrid: Polifemo, 2015).

${ }^{5}$ Sobre los Lemos véanse los estudios de Valentina Favarò, Carriere in movimento. Francisco Ruiz de Castro e la monarchia di Filippo III (Palermo: Mediterranea, 2013); Gobernar con prudencia. Los Lemos, estrategias familiares y servicio al Rey (siglo XVII) (Murcia: Universidad de Murcia, 2016). Sobre el papel de esa familia en la facción de Lerma, Giuseppe Mrozek Eliszezynski, "Service to the King and Loyalty to the Duke. The Castro Family in the Faction of the Duke of Lerma", en The Secret Mechanisms of Courts: Factions in Early Modern Europe, eds. Rubén González Cuerva, Valentina Caldari, Librosdelacorte.es, Monográfico 2 (2015), 68-79.

${ }^{6}$ Para más detalles, Giuseppe Mrozek Eliszezynski, "Un heredero que no está a la altura. El duque de Uceda y el fin del gobierno de los Sandoval", en Hijas e hijos de validos. Familia, género y política en la España del siglo XVII, ed. Rafael Valladares (Valencia: Albatros, 2018), 95-107.

7 Véase por ejemplo en Giovanni Muto, "«Mutation di corte, novità di ordini, nova pratica di servitori»: la «privanza» nella trattatistica politica spagnola e napoletana della prima età moderna”, en 
que estaban divididas, en los últimos años, la facción y la familia de los Sandoval (uno leal a Lerma, el otro cercano a Uceda). ${ }^{8}$ De esta guisa, Lemos y Osuna gobernaron el reino de Nápoles en manera opuesta, y si una parte de la aristocracia se inclinó hacia uno, necesariamente se opuso al otro, y viceversa. Así, se permitió a algunos nobles frontalmente contrarios al gobierno de Lemos que enviaran un embajador a Madrid para quejarse ante el rey de las acciones del virrey; estos mismos se contaron más tarde entre los partidarios de Osuna: me refiero a Carlo Caracciolo (llamado por Lemos "la bestia"), a Marino Caracciolo, príncipe de Avellino ("gran mentecato"), a los príncipes de la Riccia y de Conca y al duque de Bovino. ${ }^{9}$ Por el contrario, los que habían apoyado al gobierno (tradicionalmente considerado reformador) del conde de Lemos llevaron a cabo una feroz oposición contra el gobierno del duque de Osuna, que se caracterizó más por su falta de escrúpulos (especialmente en la política exterior) y sus acuciantes demandas económicas y militares. Entre los instigadores de los disturbios que estallaron en Nápoles en 1620 y que provocaron el retorno forzado del mismo Osuna a Madrid, se encontraban familias más bien cercanas a Lemos, como los Filomarino, los Brancaccio, algunas ramas de los Caracciolo y los Acquaviva. ${ }^{10}$

La situación política que acabamos de describir se confirma fielmente por el progreso de los Parlamentos en los años de Lerma. En las tres sesiones convocadas por el conde de Benavente, en 1604, 1607 y 1609, ${ }^{11}$ las solicitudes de donativos ordinarios presentadas por el virrey fueron fácil y rápidamente aceptadas. La ausencia de voces críticas fue casi total, tanto en la recogida de los votos para la aprobación del donativo como durante el trabajo de la Deputazione para formular las gracias que se pedían al rey y al virrey. Los regalos y ofertas al soberano, además del donativo, y las peticiones de que se confirmase a Benavente en su cargo constituyen una señal más de una aristocracia sustancialmente compacta, al menos en apariencia, en el apoyo al virrey

Con la ragione e col cuore. Studi dedicati a Carlo Capra, eds. Stefano Levati y Marco Meriggi (Milán: FrancoAngeli, 2008) 139-182. La contraposición entre el "reformador" Lemos y el "revolucionario" Osuna ha sido recurrente en mucha historiografía, como es evidente en Giuseppe Galasso, "Le riforme del conte di Lemos e le finanze napoletane nella prima metà del Seicento", en Id., Mezzogiorno medievale e moderno (Turín: Einaudi, 1965), 199-231; en Giuseppe Coniglio, I viceré spagnoli di Napoli (Nápoles: Fausto Fiorentino, 1967); o también en Rosario Villari, La rivolta antispagnola a Napoli. Le origini (15851647) (Roma-Bari: Laterza, 1967).

8 Antonio Feros, El Duque de Lerma. Realeza y privanza en la España de Felipe III (Madrid: Marcial Pons, 2002); Patrick Williams, The great favourite: the Duke of Lerma and the court and government of Philip III of Spain, 1598-1621 (Manchester-Nueva York: Manchester University Press, 2006); Alfredo Alvar Ezquerra, El Duque de Lerma. Corrupción y desmoralización en la España del siglo XVII (Madrid: La Esfera de los Libros, 2010); Mrozek Eliszezynski, Bajo acusación; Daniel Galván Desvaux, Felipe IV y la defensa del valimiento. El proceso contra el duque de Uceda (Valladolid: Ediciones Universidad de Valladolid, 2016).

${ }^{9}$ Isabel Enciso Alonso-Muñumer: Nobleza, poder y mecenazgo en tiempos de Felipe III. Nápoles y el conde de Lemos (Madrid: Actas, 2007).

${ }^{10}$ Benigno, L'ombra del re, 45-65; Luis Linde, Don Pedro Girón, duque de Osuna: la hegemonía española en Europa a comien zos del siglo XVII (Madrid: Encuentro, 2005), 189-203; Mrozek Eliszezynski, Bajo acusación, 261-278, 363-400; Giuseppe Mrozek Eliszezynski, Ascanio Filomarino. Nobiltà, Chiesa e potere nell'Italia del Seicento (Roma: Viella, 2017), 24-26.

${ }^{11}$ En 1604 se convocaron dos Parlamentos, uno extraordinario en junio y uno ordinario en diciembre: D’Agostino, Parlamento e società nel Regno di Napoli. 
y sus políticas. ${ }^{12}$ Por su parte, se llevaron a cabo sesiones mucho más animadas en los años de Lemos $(1611,1613,1615,1616)^{13}$ y Osuna $(1617,1619)$. Es muy significativo el hecho de que Giulio Cesare Di Capua Pacecco, príncipe de Conca y gran almirante del reino, fuese parte del grupo de grandes aristócratas que se opuso a la política de Lemos: de hecho, Conca fue durante décadas el primer aristócrata en tomar la palabra en el Parlamento, dar su voto y hacer la propuesta respecto a la cual el resto de la asamblea era llamado a expresarse y que, en la mayoría de los casos, terminaba por aprobar. En los años de Lemos las divisiones no fueron excesivas y la aristocracia se puso compactamente a favor de políticas controvertidas, tales como el pago de un donativo extraordinario de 1.200.000 ducados en cuatro años para que la autoridad real no procediera a una nueva numeración de los fuegos. ${ }^{14}$ Además, la asamblea pidió, como era costumbre, que el virrey fuera confirmado por el rey en su cargo. ${ }^{15}$ Muy diferente fue la situación en los Parlamentos convocados por el duque de Osuna, tanto el de 1617 como especialmente el segundo, de $1619 .{ }^{16}$ En 1617, la asamblea votó a favor de un don particular de 50.000 ducados para el duque de Uceda, gran protector de Osuna en la corte, con la petición explícita al agente del virrey, Francisco de Quevedo, de encomendar a Uceda, ya indicado como nuevo valido de Felipe III, la

\footnotetext{
12 BSNSP XXIV.A.4, Parlamento G.n.ale convocato dall'ecc.za del s.r Conte de Benavente per il Donativo ordinario del p.nte anno 1604. Alla M.tà di Filippo 3 N.S. a 18 xbre 1604, ff. 1-29v; Parlamento G.n.le convocato dall'ecc. a del sig.r Conte di Benavente per il donativo ordinario dell'anno presente 1606 trasferito ut infra alla maestà del Re Filippo 3, ff. 38-62v; Parlamento G.n.ale convocato dall'ecc.a del s.r Conte di Benavente per lo donativo ordin.o del p.nte anno 1608 alla Maestà di Filippo 3 N.S. e trasferito nel p.nte anno 1609, ff. 64v-83v.

${ }^{13}$ Los fuegos u hogares eran la unidad de recaudación fiscal. Además del Parlamento ordinario de 1615, Lemos convocó uno extraordinario en 1616 para aprobar un donativo que se asignaría al príncipe y futuro rey Felipe IV, recién casado: BSNSP XXIV.A.4, Parlamento gratioso per donatione di d.ti $200 \mathrm{~m}$ all'Altezza del Prencipe di Spagna per causa del suo casamento, ff. 177v-187r.

${ }^{14}$ Sobre las complejas motivaciones financieras, fiscales y políticas que empujaron a los nobles napolitanos a oponerse a la nueva numeración de los fuegos y a renovar, cada cuatro años y durante más de veinte años, el consiguiente donativo extraordinario de 1.200.000 ducados, véase la bibliografía mencionada en estas páginas y también Giovanni Muto, Le finanze pubbliche napoletane tra riforme e restaurazione (1520-1634) (Nápoles: Edizioni Scientifiche Italiane, 1980).

15 BSNSP XXIV.A.4, Parlamento G.n.le convocato dall'ecc. a del s.r Conte di Lemos per lo donativo del p.nte anno 1611 alla M.tà di Filippo 3 N.S., ff. 87v-120r; Parlamento G.n.le convocato dal s.r D. Pedro Fernandez de Castro Conte di Lemos per lo donativo da farsi alla M.tà di Filippo 3 N.S. sotto il di 8 di Giugno 1613, ff. 120r-141r; Parlamento G.n.ale per il donativo alla Maestà del re Filippo 3 N.S. convocato dall'ecc.a del s.r Conte de Lemos viceré di questo Regno sotto li 7 febr.o 1615, ff. 141v-172r. En el Parlamento de 1615, ya habían llegado a Nápoles las noticias del nombramiento de Lemos como nuevo presidente del Consejo de Italia y de su consiguiente regreso a Madrid. El príncipe de Conca propuso despedir a Lemos con un regalo final de 30.000 escudos de oro, además de solicitar a Felipe III que recompensase aún más al conde por sus méritos y felicitar al soberano por la elección de un personaje de tal estatura para la presidencia del Consejo de Italia.

${ }^{16}$ BSNSP XXIV.A.5, Parlamento G.n.ale convocato dall'ecc.mo s.r Duca d'Ossuna viceré di questo Regno p. lo donativo da farsi al Re Filippo 3 N.S. a 18 febr.o 1617, ff. 3r-67v; Parlamento G.n.ale convocato a 20 di feb.ro 1619 dall'ecc.mo s.r Duca d'Ossuna viceré in questo Regno di Napoli p. l'ord.o dono a S.M. di un milione, e $200 \mathrm{~m}$ doc.ti e per un altro d'altrettanta summa per la prorogat.e dell'annovero del Regno, ff. 68r-125v.
} 
defensa de los intereses del reino. ${ }^{17}$ En 1619, a pesar de la proximidad a Osuna de grandes personajes que se habían opuesto a Lemos, tales como los príncipes de Avellino y Conca, la propuesta formulada por este último fue aprobada en lo que respectaba a los dos donativos, ordinario y extraordinario. Sin embargo, se rechazó la sugerencia de no pedir nuevas gracias al rey y también la identidad de la persona que se debía enviar a Madrid como representante del reino. Conca presentó la tradicional solicitud al rey de que se confirmara el mandato del virrey para un segundo mandato trienal, pero no fue aprobada en la asamblea. Muy al contrario, en los meses siguientes, los Seggi de la capital enviaron un representante propio, fray Lorenzo Brindisi, para defender su causa y obtener la remoción de Osuna. ${ }^{18}$

Con la muerte de Felipe III en 1621 y el consiguiente fin del dominio de la familia Sandoval, comenzó la fase de la historia de la Monarquía española caracterizada por la fuerza y carisma del conde-duque de Olivares. Del mismo modo que había hecho el duque de Lerma, también el favorito del joven rey Felipe IV procuró monopolizar para él, sus parientes y aliados todos los puestos de poder, tanto en Madrid como en los territorios que componían la Monarquía. ${ }^{19}$ Nápoles no fue una excepción, y durante los más de veinte años de gobierno del conde-duque (1621-1643) se alternaron varios virreyes vinculados por relaciones de alianza más o menos duraderas con el valido. Entre ellos, fue particular la situación de Antonio Álvarez de Toledo, V duque de Alba: virrey durante siete años (1622-1629), era uno de los exponentes de la nobleza castellana más antigua y prestigiosa; entró abiertamente en conflicto con Olivares en los años siguientes, decidiendo polémicamente abandonar la corte y el servicio directo a la persona del rey (la llamada "huelga de los grandes"). Durante el gobierno napolitano de Alba, fue muy significativo el Parlamento de 1628, en el que se pueden ver dentro de la nobleza napolitana algunas divisiones destinadas a repetirse en años posteriores. ${ }^{20}$ Con una participación especialmente baja (solo 35 votantes, aunque gracias a procuraciones llegaron a manifestarse 336 votos), esta edición del Parlamento fue dominada por hombres cercanos al entonces virrey, a saber, el marqués de San Giuliano, el regente Diego López y el consejero Andrea Di Gennaro (estos dos últimos eran juristas). Además del donativo ordinario de 1.200.000 ducados, fue aprobado uno extraordinario de 300.000 ducados al año durante cuatro años, que hizo aún más pesada una carga fiscal ya difícil de soportar para los súbditos napolitanos del Rey Católico. Durante la discusión, la petición del virrey, expresada una vez más por el príncipe de Conca, fue finalmente aceptada, pero las voces críticas no faltaron.

17 Íbidem, ff. 8v-9r. La ofrenda de estos dones constituyó uno de los pilares de los juicios a los que Uceda y Osuna fueron sometidos después de la muerte de Felipe III: Mrozek Eliszezynski, Bajo acusación; Galván Desvaux, Felipe IV y la defensa del valimiento.

${ }^{18}$ Para más detalles, Mrozek Eliszezynski, Bajo acusación, 276.

${ }^{19} \mathrm{John}$ H. Elliott, The Count-Duke of Olivares. The statesman in an age of decline (New Haven-Londres: Yale University Press, 1986); Benigno, L'ombra del re, 118-145. Sobre el personaje véase ahora el estudio de Manuel Rivero Rodríguez, El conde duque de Olivares. La búsqueda de la privanza perfecta (Madrid: Polifemo, 2017).

${ }^{20}$ BNN, Brancacciano V.B.7, Parlamenti e grazie della Città di Napoli. Fatti storici nella stessa avvenuti (1554-1642), vol. 6, ff. 65v-127v; D’Agostino, Parlamento e società, 46-120. 
En ellas, más que la preocupación por las condiciones de vida de sus vasallos, se pueden apreciar exactamente las divisiones políticas dentro de la aristocracia del reino: entre los que se adhirieron al proyecto de Olivares y apoyaron a una mayor contribución del reino de Nápoles para las campañas de guerra de la Monarquía, y aquellos que se opusieron a esta estrategia y se negaron a exprimir a sus súbditos para financiar guerras consideradas ajenas a su estricto interés. Entre los primeros, a pesar de la diversidad de opiniones, estaban el príncipe de Santo Buono; Troiano Spinelli, príncipe del Oliveto; Ferrante Spinelli, príncipe de Tarsia; Fabio Carafa, príncipe de Colobraro; el duque de Caivano y muchos otros, incluidos barones y juristas. Entre los que se oponían, a su vez divididos por diferentes intereses y por nada deseosos de unir sus fuerzas contra el virrey, estaban Giovanni di Guevara, duque de Bovino; el príncipe de la Roccella; Pasquale Caracciolo, marqués de Macchiagodena; Michele Cavaniglia, duque de San Giovanni; y Giovan Battista Manso, marqués de Villa. En general, y aunque al principio no siempre fuese válido, las familias que habían estado cerca del conde de Lemos y del duque de Lerma se encontraron en la oposición durante los años de Olivares: es el caso, por ejemplo, de los Filomarino, representados en el Parlamento de 1628 por el duque de Perdifumo. ${ }^{21}$ Entre los oponentes del virrey, unidos no por casualidad por el hecho de que ninguno de ellos fue parte de la Diputación encargada de proponer las gracias, estaba también Giovanni Girolamo II Acquaviva, conde de Conversano, un poderoso y temido aristócrata que se contó entre los protagonistas de los años que precedieron a la revuelta de $1647-48 .^{22}$

La política de Olivares en Nápoles, sin embargo, fue expresada principalmente por dos virreyes: el conde de Monterrey (1631-1637) y el duque de Medina de las Torres (1637-1644). ${ }^{23}$ Con ellos se incrementaron en proporción directa tanto la presión fiscal sobre el reino (y con ella la contribución de hombres y armas a las guerras de España en Europa) como el descontento de una parte cada vez mayor de la aristocracia del reino. En el Parlamento de 1642, la tensión alcanzó el nivel más alto. Una vez más, gracias al sistema de las procuraciones fueron aprobados los donativos -ordinario y extraordinario- propuestos en nombre de Medina de las Torres por Tiberio Carafa, príncipe de Bisignano. Contó con el apoyo de personajes de la talla de Troiano Spinelli, príncipe del Oliveto (que ya había estado en posiciones lealistas en el Parlamento de 1628), o los príncipes de Santo Mango y Leporano. Pero también hubo príncipes que se opusieron, como Giuseppe Caracciolo, príncipe de Atena, así como personas que ya habían mostrado en 1628 su oposición a la política olivarista: el conde

${ }^{21}$ BNN, Brancacciano V.B.7, Parlamenti e grazie della Città di Napoli. Fatti storici nella stessa avvenuti (1554-1642), vol. 6, ff. 65v-127v, 77r.

22 Ibidem, f. 76v. Sobre Giangirolamo II Acquaviva hay una considerable bibliografía. Véanse, entre otros, Giangirolamo II Acquaviva. Un barone meridionale nella crisi del Seicento (dai memoriali di Paulo Antonio de Tarsia 1619-1665), eds. Angelantonio Spagnoletti y Giuseppe Patisso (Galatina: Congedo, 1999); Stato e baronaggio. Cultura e società nel Mezzogiorno, la Casa Acquaviva nella crisi del Seicento, ed. Caterina Lavarra (Galatina: Congedo, 2008); Aurora Martino, “Giovan Girolamo II Acquaviva d’Aragona (1604 c. - 1665). Signore feudale del Mezzogiorno spagnolo" (Tesis Doctoral, Universidad de Valladolid, 2012).

23 Benigno, L'ombra del re, 139-145; Giuseppe Galasso, Il Mezzogiorno spagnolo e austriaco, en Storia d'Italia, ed. Giuseppe Galasso (Turín: Einaudi, 2006), vol. XV, t. III, 1622-1734, 83-245; Rosario Villari, Un sogno di libertà. Napoli nel declino di un impero, 1585-1648 (Milán: Bruno Mondadori, 2012), 165-297. 
de Conversano, el marqués de Macchiagodena o la familia Filomarino (esta vez representada por el príncipe de la Rocca, Francesco Filomarino). ${ }^{24}$ No se puede olvidar tampoco a Diomede Carafa, duque de Maddaloni, protagonista de muchos acontecimientos de esos años y abiertamente hostil a la política virreinal. ${ }^{25}$ En la Diputación de las gracias de aquel Parlamento estaban como siempre personas que, por lo menos, no eran hostiles al virrey; allí encontraron un espacio otros protagonistas destinados a desempeñar un papel clave en los años siguientes: se piense en Carlo Spinelli, hermano del príncipe de Tarsia, uno de los nobles más odiados por la plebe de Nápoles; o en el barón sin título Giovanni Serio San Felice, que fue decapitado por los rebeldes en julio de 1647; o incluso en Andrea Naclerio y en el doctor Fasano, protagonistas por parte popular en la revuelta. ${ }^{26}$

Los acontecimientos entre el 7 de julio de 1647 y el 6 de abril de 1648 fueron impredecibles en muchas maneras, y los bandos que el día antes era fácil imaginar solo se hicieron realidad parcialmente. La revuelta tomó una naturaleza antinobiliaria y filopopular que pronto dejó a un lado a esas figuras aristocráticas que habían aspirado a hacerse líderes de los rebeldes, tal vez con el apoyo de la Monarquía francesa. ${ }^{27}$ Así fue que el conde de Conversano y el duque de Maddaloni se encontraron luchando en las provincias del reino junto a nobles, como el príncipe de Bisignano, a los que se habían opuesto en años anteriores. Pero incluso si su ferocidad y la contribución de sus ejércitos fueron fundamentales para sofocar la revuelta en Apulia y otras zonas del sur de Italia, el poder español no se olvidó de sus acciones en los años precedentes a la revuelta, privándoles gradualmente de parte de su poder e influencia. ${ }^{28}$ Los

${ }^{24}$ BNN, Brancacciano V.B.7, Parlamenti e grazie della Città di Napoli. Fatti storici nella stessa avvenuti (1554-1642), vol. 6, ff. 65v-127v, ff. 70v-81v; Giuseppe Carignani, "L'ultimo parlamento generale del Regno di Napoli nel 1642”, Archivio storico per le province napoletane 8 (1883): 34-57.

25 Alfred von Reumont, The Carafas of Maddaloni: Naples under Spanish Dominion (Londres: H.G. Bohn, 1854); Carla Russo, "Carafa, Diomede", DBI 19 (1976): 533-535; Dandolo, Sabatini, Lo Stato feudale dei Carafa di Maddaloni; I Carafa di Maddaloni e la feudalità napoletana nel Mezzogiorno spagnolo: atti in memoria di S.E. Mons. Pietro Farina, eds. Francesco Dandolo y Gaetano Sabatini (Caserta: Edizioni Saletta dell'Uva, 2013); Mrozek Eliszezynski, Ascanio Filomarino.

${ }^{26}$ BNN, Brancacciano V.B.9, Parlamenti e grazie della Città di Napoli. Fatti storici nella stessa avvenuti (1554-1642), vol. 9; Carignani, L'ultimo parlamento generale del Regno di Napoli nel 1642, 38-40, 44-45.

${ }^{27}$ Dentro de la muy amplia historiografía sobre la revuelta de 1647-48, y además de las obras mencionadas anteriormente, véanse también: Aurelio Musi, La rivolta di Masaniello nella scena politica barocca (Nápoles: Guida, 1989); Francesco Benigno, "Il mistero di Masaniello", en Id., Specchi della rivoluzione. Conflitto e identità politica nell'Europa moderna (Roma: Donzelli, 1999), 199-285, y en particular el capítulo "Una nobiltà inconfidente", 256-262; Alain Hugon, Naples insurgé 1647-48. De l'événement à la memoire (Rennes: Presses universitaires de Rennes, 2011).

${ }^{28}$ Sobre la difusión de la revuelta en las varias provincias del reino hay una rica bibliografía, dentro de la cual señalamos: Ludovico Pepe, Nardò e Terra d'Otranto nei moti del 1647-48 (Trani: Vecchi, 1894); Francesco Andreu, Francesco Surgente vescovo di Monopoli e i moti masanelliani del 1647-48, in Studi di storia pugliese in onore di G. Chiarelli (Galatina: Congedo, 1974), vol. III, 219-308; Giovanni Celoro Parascandolo, Cronache inedite della rivoluzione di Masaniello (Nápoles: Nuove Edizioni, 1985); Giuseppe Foscari, La gran machina della sollevatione. Due città e un capopopolo nella rivolta di Masaniello (1647-1648) (Marsicovetere: Ipermedium, 2015). Sobre las vicisitudes de parte de la nobleza después de la revuelta, véase en cambio el estudio clásico de Giuseppe Galasso, Napoli spagnola dopo Masaniello. Politica, cultura, società, 2 vols. (Florencia: Sansoni, 1982). 
Filomarino también se encontraban en una posición crítica en relación con el gobierno de Olivares, pero a la vez opuestos por antiguas enemistades a otras familias del mismo bando (en particular a los Carafa de Maddaloni). Ante estas condiciones, tuvieron un comportamiento deliberadamente ambiguo durante el levantamiento. Encabezados por su pariente el cardenal y arzobispo de Nápoles Ascanio Filomarino, el príncipe de la Rocca y el duque de Perdifumo fueron por mucho tiempo una parte importante del frente rebelde; especialmente el príncipe de la Rocca recibió del propio Masaniello el cargo de grassiere (prefecto de la anona) de la ciudad. Pero luego, cuando se hizo evidente que los acontecimientos estaban empeorando para los rebeldes, se situaron entre los más activos en las conversaciones secretas con don Juan de Austria, el hijo natural de Felipe IV que había llegado al mando de la flota española en octubre de 1647. En los años siguientes, los mismos Filomarino se jactaron por mucho tiempo de que, sin su contribución, la ciudad nunca se habría recuperado tan rápida y fácilmente como sucedió el 6 de abril de 1648, cuando las tropas españolas no encontraron prácticamente ninguna resistencia en su entrada en la ciudad. ${ }^{29}$

En el periodo siguiente a la revuelta, que coincide con el gobierno del nuevo virrey, el conde de Oñate, el debate sobre las responsabilidades de los recientes acontecimientos fue vivo y dramático. ${ }^{30}$ En Madrid había comenzado el gobierno del nuevo favorito de Felipe IV, don Luis de Haro, aunque Oñate no puede definirse estrictamente como un verdadero aliado o cliente del valido. ${ }^{31}$ No solo los líderes populares más importantes pagaron con sus vidas por su traición al rey de España (como Gennaro Annese), sino que también muchos nobles fueron duramente castigados por el virrey por el comportamiento que habían tenido antes y durante la revuelta. Consciente de lo que había ocurrido en el Parlamento de 1642 y de la presencia de tantos protagonistas del levantamiento en esa sesión, el gobierno español decidió no volver a convocar el Parlamento y que los Seggi de Nápoles fueran la institución encargada de decidir para el futuro la aprobación de los impuestos directos sobre los súbditos del reino. Algunos de los nobles que habían conspirado contra el poder español siguieron dando problemas al virrey, como en los famosos casos de Tommaso de Saboya, que llegó a Nápoles al mando de una flota francesa el 4 de agosto de 1648 pero fue rápidamente derrotado, ${ }^{32}$ y de Andrea d'Avalos, príncipe de Montesarchio, líder de una controvertida conspiración nobiliaria filofrancesa. ${ }^{33}$ Es verdad que Oñate fue capaz de ejercer un control más estricto sobre el pueblo de

\footnotetext{
${ }^{29}$ Mrozek Eliszezynski, Ascanio Filomarino, 160-168.

${ }^{30}$ Giuseppe Mrozek Eliszezynski, "Le responsabilità della rivolta. Le accuse del viceré Oñate e le risposte del cardinal Filomarino (1648-1653)", Dimensioni e problemi della ricerca storica, I (2017), 119-151.

${ }^{31}$ Sobre la corte de Madrid durante el gobierno de Luis de Haro y sobre las relaciones entre el favorito de Felipe IV y los principales protagonistas de la lucha política cortesana, véanse El mundo de un valido. Don Luis de Haro y Guzmán y su entorno, 1643-1661, ed. Rafael Valladares (Madrid: Marcial Pons, 2016); Alistair Malcolm, Royal Favouritism and the Governing Elite of the Spanish Monarchy, 1640-1665 (Oxford: Oxford University Press, 2017).

32 Giuseppe Carignani, "Tentativi di Tommaso di Savoia per impadronirsi del trono di Napoli", Archivio Storico per le Province Napoletane 6 (1881): 663-731.

33 Michelangelo Schipa, "La congiura del principe di Montesarchio", Archivio Storico per le Province Napoletane 43 (1918): 271-296; 44 (1919): 191-226; 45 (1920): 251-279.
} 
Nápoles confiando la tarea de Eletto del Popolo (representante del Seggio popular) a un hombre leal a él como Giuseppe Volturale; no obstante, no es menos cierto que la parte de la nobleza, feudal y urbana, que había demostrado su lealtad durante la revuelta fue recompensada por el virrey y participó plenamente en el gran proyecto de renovación cultural y urbana que Oñate quería. Grandes aristócratas como el duque de Monteleone, el marqués de Fuscaldo, el marqués del Oliveto y el duque de Sasso se convirtieron en influyentes asesores de Oñate, pero en realidad la mayoría de la aristocracia del reino se volvió contra el virrey hasta conseguir finalmente su regreso a Madrid en 1653. Pesaron en su contra la actitud de puño de hierro que mostró hacia muchos nobles después de la revuelta, el no haber restaurado el sistema de privilegios (también al nivel de los impuestos) que había sido eliminado por los rebeldes y el apoyo demasiado evidente que el virrey brindó a la clase emergente de los juristas, los llamados togati. ${ }^{34}$

Entre los que más presionaron para reemplazar a Oñate estuvo también el cardenal Filomarino, miembro de una familia opuesta a la política de Olivares y entre las que más habían procurado cambiar las relaciones de poder del reino. ${ }^{35}$ Filomarino representa, junto con su familia, el ejemplo ideal de lo que he intentado mostrar en extrema síntesis en esta intervención: estaba vinculado al conde de Conversano por matrimonio de parientes comunes y era odiado por muchos otros nobles como el duque de Maddaloni o el marqués del Torello (con el que estuvo involucrado en una dramática disputa pública en noviembre de 1648). ${ }^{36}$ A partir de diferentes períodos y elementos de reflexión, he intentado poner de relieve cómo la aristocracia napolitana se fue recolocando durante la primera mitad del siglo XVII según los virreyes que llegaban a Nápoles y los lazos faccionales y clientelares de los que estos eran expresión. Algunas familias mantuvieron una línea de acción y pensamiento consistente con el paso de los años, mientras que otras cambiaron sus intereses y proyectos. La participación en la política cultural deseada por el virrey o el intercambio mutuo de dones fueron formas de establecer o fortalecer lazos y alianzas. A través del estudio de la aristocracia napolitana en los años del valimiento es posible, por lo tanto, entender mejor la evolución de la lucha política en el reino de Nápoles. El análisis de los Parlamentos es solo una de las herramientas disponibles para abordar un tema de tan largo alcance y de tal importancia en la historia de la Monarquía de los Habsburgo y de la Italia española.

\footnotetext{
34 Galasso, Il Mezzogiorno spagnolo e austriaco, t. III, 1622-1734, 519-552; Mrozek Eliszezynski, Ascanio Filomarino, 169-205. Para una mirada general a la biografía del conde de Oñate, véase Ana Minguito Palomares, Nápoles y el virrey conde de Oñate. La estrategia del poder y el resurgir del reino (1648-1653) (Madrid: Sílex, 2011). En cuanto al ascenso de los togati, la referencia es a los estudios de Pier Luigi Rovito, $L a$ Respublica dei togati. Giuristi e società nella Napoli del Seicento (Nápoles: Jovene, 1981); "La rivoluzione costituzionale di Napoli (1647-48)", Rivista storica italiana 98 (1986): 367-462; Il viceregno spagnolo di Napoli. Ordinamento, Istituzioni, Culture di governo (Nápoles: Arte Tipografica, 2003).

35 Aurelio Musi, Introdurione, en Il Regno di Napoli nell'età di Filippo IV, 11-33; Sodano, Le aristocrazie napoletane.

${ }^{36}$ Mrozek Eliszezynski, Ascanio Filomarino, 181-183.
} 


\section{REFERENCIAS BIBLIOGRÁFICAS}

Biagio Aldimari, Memorie historiche di diverse famiglie nobili, cosi napolitane come forastiere, cosi vive come spente, con le loro arme; e con un trattato dell'arme in generale (Nápoles: Raillard, 1691).

Alfredo Alvar Ezquerra, El Duque de Lerma. Corrupción y desmoralización en la España del siglo XVII (Madrid: La Esfera de los Libros, 2010).

Scipione Ammirato, Delle famiglie nobili napoletane (Florencia: Marescotti, 1580-1651).

Francesco Andreu, "Francesco Surgente vescovo di Monopoli e i moti masanelliani del 1647-48", en Studi di storia pugliese in onore di G. Chiarelli (Galatina: Congedo, 1974), vol. III, 219-308.

Francesco Benigno, "Persistere, resistere: Parlamenti italiani e Monarchia degli Asburgo", en Id., Favoriti e ribelli. Stili della politica barocca (Roma: Bulzoni, 2011), 147-163.

Francesco Benigno, "Una nobiltà inconfidente", en Id., Specchi della rivoluzione. Conflitto e identità politica nell'Europa moderna (Roma: Donzelli, 1999), 256-262.

Francesco Benigno, L'ombra del re. Ministri e lotta politica nella Spagna del Seicento (Venecia: Marsilio, 1992).

Filiberto Campanile, L'Armi overo insegne de’ Napoli (Nápoles: Longo, 1610).

Berardo Candida Gonzaga, Memorie delle famiglie nobili delle province meridionali d'Italia, 6 vols. (Nápoles: De Angelis e figlio, 1875-1879).

Francesco Capecelatro, Origine della città e delle famiglie nobili di Napoli (Nápoles: Gravier, 1769).

Francesco Caracciolo, "Il Parlamento del Regno di Napoli durante la dominazione spagnola", Quaderni contemporanei 4 (1971): 21-58.

Giuseppe Carignani, "L’ultimo parlamento generale del Regno di Napoli nel 1642”, Archivio storico per le province napoletane 8 (1883): 34-57.

Giuseppe Carignani, "Tentativi di Tommaso di Savoia per impadronirsi del trono di Napoli”, Archivio Storico per le Province Napoletane 6 (1881): 663-731.

Giovanni Celoro Parascandolo, Cronache inedite della rivolurione di Masaniello (Nápoles: Nuove Edizioni, 1985). 
Giuseppe Coniglio, I viceré spagnoli di Napoli (Nápoles: Fausto Fiorentino, 1967).

Benedetto Croce, Storia del Regno di Napoli (Bari: Laterza, 1925).

Elena Croce, "I parlamenti napoletani sotto la dominazione spagnola", Archivio storico per le province napoletane 61 (1936): 341-379.

Guido D’Agostino, Parlamento e società nel Regno di Napoli, secoli XV-XVII (Nápoles: Guida, 1979).

Francesco Dandolo y Gaetano Sabatini, Lo Stato feudale dei Carafa di Maddaloni. Genesi e amministrazione di un ducato nel regno di Napoli (secc. XV-XVIII) (Nápoles: Giannini, 2009).

Francesco Dandolo y Gaetano Sabatini (eds.), I Carafa di Maddaloni e la feudalità napoletana nel Merzogiorno spagnolo: atti in memoria di S.E. Mons. Pietro Farina (Caserta: Edizioni Saletta dell'Uva, 2013).

Carlo De Lellis, Discorsi delle famiglie nobili del Regno di Napoli (Nápoles 1654-1671).

John H. Elliott, The Count-Duke of Olivares. The statesman in an age of decline (New HavenLondres: Yale University Press, 1986).

Isabel Enciso Alonso-Muñumer: Nobleza, podery mecenazgo en tiempos de Felipe III. Nápoles $y$ el conde de Lemos (Madrid: Actas, 2007).

Valentina Favarò, Carriere in movimento. Francisco Ruiz de Castro e la monarchia di Filippo III (Palermo: Mediterranea, 2013).

Valentina Favarò, Gobernar con prudencia. Los Lemos, estrategias familiares y servicio al Rey (siglo XVII) (Murcia: Universidad de Murcia, 2016).

Antonio Feros, El Duque de Lerma. Realeza y privanza en la España de Felipe III (Madrid: Marcial Pons, 2002).

Giuseppe Foscari, La gran machina della sollevatione. Due città e un capopopolo nella rivolta di Masaniello (1647-1648) (Marsicovetere: Ipermedium, 2015).

Giuseppe Galasso, "Le riforme del conte di Lemos e le finanze napoletane nella prima metà del Seicento", en Id., Mezzogiorno medievale e moderno (Turín: Einaudi, 1965), 199-231. 
Giuseppe Galasso, Il Merzogiorno spagnolo e austriaco, en Storia d'Italia, ed. Giuseppe Galasso (Turín: Einaudi, 2006), vol. XV, t. II, 1494-1622.

Giuseppe Galasso, Il Mezzogiorno spagnolo e austriaco, en Storia d'Italia, ed. Giuseppe Galasso (Turín: Einaudi, 2006), vol. XV, t. III, 1622-1734.

Giuseppe Galasso, Mezzogiorno medievale e moderno (Turín: Einaudi, 1965).

Giuseppe Galasso, Napoli spagnola dopo Masaniello. Politica, cultura, società, 2 vols. (Florencia: Sansoni, 1982).

Daniel Galván Desvaux, Felipe IV y la defensa del valimiento. El proceso contra el duque de Uceda (Valladolid: Ediciones Universidad de Valladolid, 2016).

Alain Hugon, Naples insurgé 1647-48. De l'événement à la memoire (Rennes: Presses universitaires de Rennes, 2011).

Caterina Lavarra (ed.), Stato e baronaggio. Cultura e società nel Mezzogiorno, la Casa Acquaviva nella crisi del Seicento (Galatina: Congedo, 2008).

Luis Linde, Don Pedro Girón, duque de Osuna: la hegemonía española en Europa a comienzos del siglo XVII (Madrid: Encuentro, 2005).

Alistair Malcolm, Royal Favouritism and the Governing Elite of the Spanish Monarchy, 16401665 (Oxford: Oxford University Press, 2017).

Antonio Marongiu, Il Parlamento in Italia nel medioevo e nell'età moderna (Milán: Giuffrè, 1962).

Aurora Martino, “Giovan Girolamo II Acquaviva d'Aragona (1604 c. - 1665). Signore feudale del Mezzogiorno spagnolo" (Tesis Doctoral, Universidad de Valladolid, 2012).

Ana Minguito Palomares, Nápoles y el virrey conde de Oñate. La estrategia del poder y el resurgir del reino (1648-1653) (Madrid: Sílex, 2011).

Giuseppe Mrozek Eliszezynski, "Service to the King and Loyalty to the Duke. The Castro Family in the Faction of the Duke of Lerma", en The Secret Mechanisms of Courts: Factions in Early Modern Europe, eds. Rubén González Cuerva, Valentina Caldari, Librosdelacorte.es, Monográfico 2 (2015): 68-79.

Giuseppe Mrozek Eliszezynski, Bajo acusación: el valimiento en el reinado de Felipe III. Procesos y discursos (Madrid: Polifemo, 2015). 
Giuseppe Mrozek Eliszezynski, Ascanio Filomarino. Nobiltà, Chiesa e potere nell'Italia del Seicento (Roma: Viella, 2017).

Giuseppe Mrozek Eliszezynski, "Le responsabilità della rivolta. Le accuse del viceré Oñate e le risposte del cardinal Filomarino (1648-1653)", Dimensioni e problemi della ricerca storica 1 (2017): 119-151.

Giuseppe Mrozek Eliszezynski, "Un heredero que no está a la altura. El duque de Uceda y el fin del gobierno de los Sandoval", en Hijas e hijos de validos. Familia, género y política en la España del siglo XVII, ed. Rafael Valladares (Valencia: Albatros, 2018), 95-107.

Aurelio Musi, La rivolta di Masaniello nella scena politica barocca (Nápoles: Guida, 1989).

Aurelio Musi, "Introduzione", en Il Regno di Napoli nell'età di Filippo IV (1621-1665), eds. Giovanni Brancaccio y Aurelio Musi (Milán: Guerini e Associati, 2014), 11-33.

Giovanni Muto, “«I segni d'honore». Le rappresentazioni delle dinamiche nobiliari a Napoli in età moderna", en Signori, patrizi, cavalieri nell'età moderna, ed. Maria Antonietta Visceglia (Roma-Bari: Laterza, 1992), 171-192.

Giovanni Muto, “《Mutation di corte, novità di ordini, nova pratica di servitori»: la «privanza» nella trattatistica politica spagnola e napoletana della prima età moderna", en Con la ragione e col cuore. Studi dedicati a Carlo Capra, eds. Stefano Levati y Marco Meriggi (Milán: FrancoAngeli, 2008), 139-182.

Giovanni Muto, Le finanze pubbliche napoletane tra riforme e restaurazione (1520-1634) (Nápoles: Edizioni Scientifiche Italiane, 1980).

Ludovico Pepe, Nardò e Terra d'Otranto nei moti del 1647-48 (Trani: Vecchi, 1894).

Giuseppe Recco, Notizie di famiglie nobili ed illustri della città e Regno di Napoli (Nápoles: Parrino, 1717).

Alfred von Reumont, The Carafas of Maddaloni: Naples under Spanish Dominion (Londres: H.G. Bohn, 1854).

Manuel Rivero Rodríguez, El conde duque de Olivares. La búsqueda de la privanza perfecta (Madrid: Polifemo, 2017).

Pier Luigi Rovito, La Respublica dei togati. Giuristi e società nella Napoli del Seicento (Nápoles: Jovene, 1981). 
Pier Luigi Rovito, "La rivoluzione costituzionale di Napoli (1647-48)", Rivista storica italiana 98 (1986): 367-462.

Pier Luigi Rovito, Il viceregno spagnolo di Napoli. Ordinamento, Istituzioni, Culture di governo (Nápoles: Arte Tipografica, 2003).

Carla Russo, "Carafa, Diomede”, DBI 19 (1976): 533-535.

Elisabetta Scarton y Francesco Senatore, Parlamenti generali a Napoli in età aragonese (Nápoles: Federico II University Press, 2018).

Michelangelo Schipa, "La congiura del principe di Montesarchio", Archivio Storico per le Province Napoletane 43 (1918): 271-296; 44 (1919): 191-226; 45 (1920): 251-279.

Giulio Sodano, "Le aristocrazie napoletane", en Il Regno di Napoli nell'età di Filippo IV (1621-1665), eds. Giovanni Brancaccio y Aurelio Musi (Milán: Guerini e Associati, 2014), 131-176.

Giulio Sodano, Da baroni del Regno a Grandi di Spagna. Gli Acquaviva d'Atri: vita aristocratica e ambiz̨onion politiche (secoli XV-XVIII) (Nápoles: Guida, 2012).

Angelantonio Spagnoletti y Giuseppe Patisso (eds.), Giangirolamo II Acquaviva. Un barone meridionale nella crisi del Seicento (dai memoriali di Paulo Antonio de Tarsia 1619-1665) (Galatina: Congedo, 1999).

Camillo Tutini, Dell'origine e fundation de' Seggi di Napoli, del tempo in che furono instituiti e della separation de' nobili dal popolo (Nápoles: Beltrano, 1644).

Rafael Valladares (ed.), El mundo de un valido. Don Luis de Haro y Guzmán y su entorno, 1643-1661 (Madrid: Marcial Pons, 2016).

Rosario Villari, La rivolta antispagnola a Napoli. Le origini (1585-1647) (Roma-Bari: Laterza, 1967).

Rosario Villari, Un sogno di libertà. Napoli nel declino di un impero, 1585-1648 (Milán: Bruno Mondadori, 2012).

Maria Antonietta Visceglia, Il bisogno di eternità. I comportamenti aristocratici a Napoli in età moderna (Nápoles: Guida, 1988).

Maria Antonietta Visceglia, Identità sociali. La nobiltà napoletana nella prima età moderna (Milán: Unicopli, 1998). 
Patrick Williams, The great favourite: the Duke of Lerma and the court and government of Philip III of Spain, 1598-1621 (Manchester-Nueva York: Manchester University Press, 2006).

Recibido: 03 de agosto de 2018 Aprobado: 21 de marzo de 2019 